\title{
Efectos del bullying en los adolescentes
}

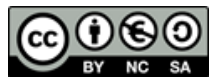

\section{Effects of bullying on teenagers}

\author{
Pablo Homero Velasteguí López. ${ }^{1}$
}

Resumen.

DOI: https://doi.org/10.33262/concienciadigital.v2i2.940

El bullying es evidente en el ámbito escolar, este tipo de violencia lo llevan a cabo los mismos alumnos ya sea entre iguales o no, sus conductas son de intimidación acoso, amenaza, burlas o insultos en esta investigación analizamos la violencia en los adolescentes pudiendo centrarnos principalmente en lo que se produce en el ámbito escolar. En primer lugar pudimos tomar en cuenta los conceptos de agresividad, violencia, y otros relacionados. A continuación, revisamos y pudimos comparar algunas investigaciones que son más destacadas acerca de la incidencia del bullying; para luego así poder reflexionar sobre las causas y circunstancias que facilitan y ayudan a mantener ese tipo de conductas. Tenemos la capacidad de sugerir algunas líneas que facilitan la actuación de distintas instancias sociales que se encaminen hacia su prevención.

Por lo general hay personas que siempre recurren a la violencia y la agresión para poder dominar a sus semejantes o para tratar de armar nuevos conflictos es muy común que en nuestro marco cultural podamos entender que las conductas violentas y agresivas no forman parte de una conducta adecuada para la convivencia diaria. También entre otras cosas las agresiones pueden conllevar a la represión de un conflicto, la sociedad también comenta que a veces la violencia aparenta ser muy sensibilizada en ciertas manifestaciones, como la violencia juvenil, o la violencia de género, etc. En concreto en las últimas décadas, los medios de comunicación han destacado las noticias relacionadas con la violencia de los adolescentes específicamente, con la que se produce en el ámbito escolar.

\footnotetext{
${ }^{1}$ Ciencia digital, Ambato, Ecuador, pablohomerovelastegui@cienciadigital.org
} 
Palabras claves: Bullying, violencia juvenil, adolescencia, prevención, escolar.

\section{Abstract.}

Bullying is evident in the school environment, this type of violence is carried out by the same students, whether among peers or not, their behaviors are intimidation, harassment, threats, ridicule or insults. In this research, we analyze violence in adolescents and can focus mainly in what is produced in the school environment. In the first place we were able to take into account the concepts of aggressiveness, violence, and other related ones. Next, we reviewed and we were able to compare some researches that are more prominent about the incidence of bullying; to then be able to reflect on the causes and circumstances that facilitate and help maintain this type of behavior. We have the capacity to suggest some lines that facilitate the action of different social instances that are directed towards their prevention.

Usually there are people who always resort to violence and aggression to dominate their peers or to try to build new conflicts is very common in our cultural framework we can understand that violent and aggressive behaviors are not part of proper behavior for daily coexistence. Also, among other things, aggressions can lead to the repression of a conflict, society also comments that sometimes violence appears to be very sensitive in certain manifestations, such as youth violence, or gender violence, etc. Specifically in recent decades, the media has highlighted the news related to adolescent violence specifically, with that which occurs in the school environment.

Keywords: Bullying, juvenile violence, adolescence, prevention, school.

\section{Introducción.}

\section{Que es el bullying.}

Según los autores (Cerezo, 2002), (Portero, 2007)\& (Casilla, 2008) el bullying es el maltrato físico, psicológico continuo que recibe un niño o un adolescente por parte de otro u otros que se comportan cruelmente con el objetivo de someterlo y asustarlo esto es para obtener un resultado favorable por parte de los acosadores o simple y sencillamente para satisfacer la necesidad de agredir a los demás es una realidad que existe en algunas escuelas o colegios desde siempre y se ha considerado un proceso normal dentro de una cultura en silencio que ayuda a su perpetuación. Hay casos que las personas recurren en numerosas ocasiones a la violencia y a la agresión, tal vez piensen que es la mejor manera de sentirse superiores a los demás pero lo que no saben es que están causando un mal a la sociedad y también están lastimando a los demás de manera física y psicológica son casos que en muchas ocasiones son suscitados en las escuelas o ya sea en los colegios, es algo que en ocasiones los adultos no están enterados porque ya sea los niños o los adolescentes no hablan por miedo hacer más lastimados de lo habitual y tienen miedo que esto conlleve a algo peor. 


\section{Criterio:}

En mi opinión el bullying es un problema muy grande en el ámbito escolar el cual perjudica a los estudiantes ya sea física o psicológicamente también nos da a entender que cuando se da el caso de bullying tienen a la opción de maltratarlos de forma física psicológica esto nos quiere decir que los maltratos son con insultos, golpes. Debemos tener en cuenta que para llevar una vida estudiantil tenemos que hablar sobre los casos suscitados para que así haya una favorable solución para que esto no conlleve a instancias peores, que en algún momento tengamos que lamentar. Siempre cuando tenemos un problema ya sea de cualquier tipo tenemos que hablar con nuestros padres para que ellos nos ayuden a solucionar o ya sería una mejor opción nos lleven a una especialista para que los resultados sean más favorables y así podamos tener mejores soluciones y poder solucionar los conflictos que se estén dando y tener buenos resultados.

\section{Violencia juvenil.}

Según los autores (Wielandt, 2005), (Rubio, 2007) \& (Morales, 2008) la violencia juvenil es el comportamiento antisocial durante la adolescencia o la combinación del múltiples factores ubicados en diferentes niveles del desarrollo humano, o también son procesos sociales o culturales que afectan de manera diferenciada a varias generaciones de jóvenes desde una muy temprana edad, también se puede decir que es un comportamiento deliberado que se ejecuta con fuerza y ímpetu generando daños físicos o emocionales a los demás seres humanos, es un acto muy violento que se realiza contra la voluntad o el gusto de la víctima, un insulto un empujón o ya sea un golpe son acciones de violencia juvenil esto suele suceder en la edad que se sitúa ente la infancia y la vida adulta aproximadamente entre los 15 a los 25 años. Aunque no existen de todas formas límites precisos de cómo podemos definir a la juventud.

No se pude decir que solo los jóvenes ocasionan violencia porque en ciertos casos los niños o personas adultas también suelen generar actos de violencia. Casi en todos los países los adolescentes, y los adultos jóvenes son tanto las principales víctimas como los principales perpetradores de la violencia.

\section{Criterio.}

En mi opinión la violencia juvenil es un muy mal comportamiento antisocial en la adolescencia, también nos quiere decir que hay factores de diferentes niveles en el maltrato al ser humano o se podría decir que es un comportamiento deliberado que se lo realiza con la fuerza generando daños físicos, emocionales a las personas que son víctimas del bullying esto suelen ser actos de violencia que son realizados contra la voluntad o el consentimiento de la víctima, nos dice que en las edades de los 11 a los 17 años es más común ver casos de bullying ya que ellos siempre quieren ser los protagonista en todas las acciones que se 
suscitan para así poder sentirse los héroes pero lo que no saben es que están ocasionando un terrible daño no son capases de darse cuenta que esto conlleva a ocasionar un terrible daño a la sociedad.

\section{Casos de bullying en Ecuador.}

Según los autores (Pando, 2012), (Cusot, 2012) \& (Aguirre, 2014) en las estadísticas realizadas en una muestra de 980 niños y adolescentes revelo que un $32 \%$ de niños y adolescentes de un total de 5 millones que hay en nuestro país, es insultado en ocasiones por sus compañeros. El 22,6\% son niños entre la edad de 6 a 11 años los cuales sufren maltratos por partes de sus compañeros de escuela, y el 20,1\% son jóvenes de 15 a 17 años. Nos dice también que el bullying o acoso escolar no es problema reciente es algo que viene ocurriendo en el transcurso de toda la vida, antes lo veían como un problema de mala conducta en los niños. Nos dice que por lo general un niño que es agredido no hable por temor a las amenazas realizadas por parte de los niños que practican este tan nombrado bullying en ese ámbito un niño que actúa como agresor se lo ve como el mejor alumno, es el más popular; mientras que el niño agredido es el más débil del aula y el más tímido. Es por eso que siente temor de contar lo que está sucediendo a los mayores, es muy importante que cuando ellos hablen sobre lo que está sucediendo los padres tomen mucha atención para que así puedan orientarlos con la ayuda de un especialista. También nos dice que algunos casos el agresor actúa por varios motivos; porque fue víctima antes, o porque necesita sentir el poder atreves del hecho de hacer daño a otros motivos porque también son agredidos en el hogar o simplemente lo hacen para alcanzar popularidad quieren ser estrellas.

Figura N 1. Maltrato entre pares según región.

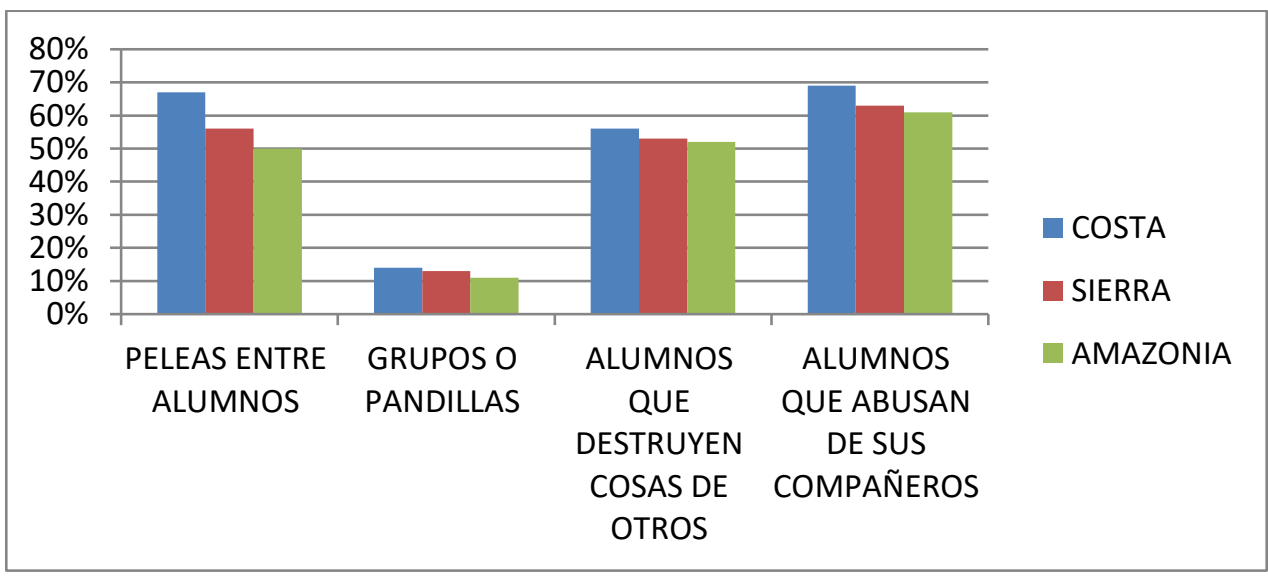

Fuente: Elaborada propia 


\section{Criterio:}

En mi opinión el bullying no es un problema reciente es algo de toda la vida antes se lo caracterizaba como una mala conducta es algo que también abarca a una sociedad es más se puede apreciar en el documento investigado que los niños y los adolescentes de nuestro país sufren con el acoso escolar es mas no solo los niños sufren de este acoso las niñas mujeres también hacen parte del maltrato escolar. Tenemos que tener muy en cuenta que el bullying no es una broma, es algo muy serio que siempre tenemos que tener presente para así poder ayudar a los niños y adolescentes a que lleven una vida estudiantil alegre y muy tranquila para que así tengan un futuro con éxito.

\section{Estadística del bullying en los adolescentes}

Según los autores (Garcia, 2010), (Sanchez, 2012) \& (Cava, 2012) El acoso escolar constituye un problema social y de salud que ha generado una alarma en los últimos años. Se puede decir que en este estudio se describe el acoso escolar y los factores relacionados entre estudiantes. La prevalencia de acoso escolar fue de 18,2\%, 10,9\%, y 4,3\% en chicos, y del $14,4 \%, 8,5 \%$, y $4,5 \%$ en chicas de segundo y cuarto de educación secundaria y segundo de bachillerato o siclos formativos de grado medio respectivamente. Los factores que se asociaron con un incremento de la probabilidad de padecer acoso fueron el estado de ánimo negativo y la conducta violenta, mientras que al tener mayor edad, el riesgo seria al consumo del alcohol, e ir a bares y discotecas se lo asocia como un acto negativo. Cada victimización entre seres iguales en el contexto escolar determinadas variables de ajustes psicosocial, como la satisfacción con la vida, la autoestima, la sintomatología depresiva y la sociedad la muestra estuvo formada por 1319 adolescentes de edades comprendidas entre los 11 y 16 años.

\section{Criterio.}

En mi opinión el bullying es un problema que se ha suscitado a nivel nacional e internacional ya que este afecta en la vida de algunos estudiantes que son víctimas de acoso ya sea en las escuelas o en los colegios los cuales son perjudicados física o psicológicamente. Las víctimas de este asedio comienzan a veces con un simple abucheo y terminar en muy pocas ocasiones con un desenlace fatal.

\section{Países con más alto bullying a nivel mundial}

Según los autores (Meglino, 2018) (Sanchez A., 2018) (Merchan, 2018) Hay varios países con altas estadísticas de bullying en los cuales los niños, niñas y adolescentes son víctimas de acoso escolar por lo que están realizando charlas atreves de la ONG Bullying sin fronteras en 18 países de América, siete de cada 10 niños son víctimas de este asedio que a veces solo comienza con un simple abucheo y a veces termina en un desenlace fatal. Nos dice que cada vez es más habitual encontrarse con alarmantes estadísticas y casos relacionados a la violencia escolar y el bullying en los medios locales e internacionales. 
Figura N 2 Países con índice más alto en Bullying

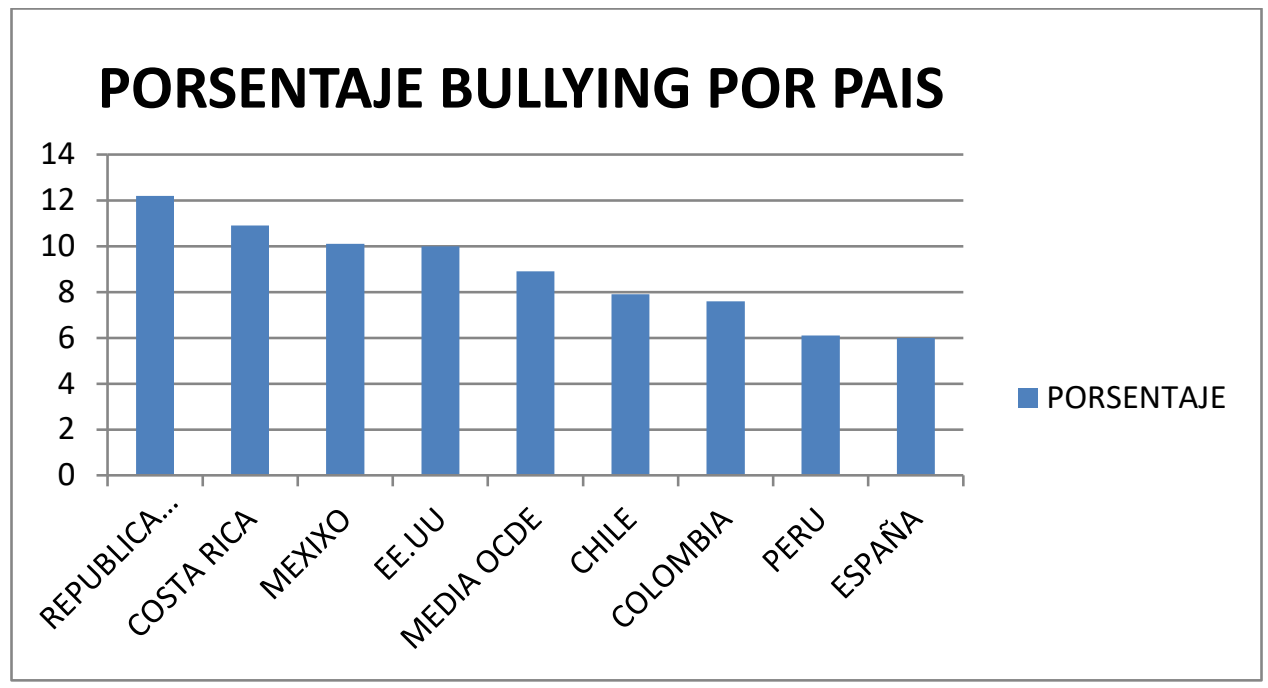

Fuente: Elaborada propia

\section{Criterio.}

En mi opinión es muy habitual encontrarse con casos de violencia escolar lo que nos dice que tenemos 19 países con un alto porcentaje de acoso escolar en lo investigado pudimos encontrar los países en los cuales encontramos la mayor estadística de acoso escolar, en lo cual debemos trabajar mucho para poder disminuir el bullying en todos estos países y que los alumnos tengan tranquilidad en sus días laborables en las escuelas y colegios.

\section{Causas del bullying.}

Según los autores (Lossi, 2015) (Aviles, 2015) (Medina, 2015) nos dice que sin importar el tipo de institución educativa, pública o privadas, los colegios representan problemas de acoso escolar. Según la Organización para la Cooperación y Desarrollo Económico (OCDE), el $85 \%$ de la violencia que ocurre en la escuela, y el $82 \%$ de los niños con discapacidad sufren bullying escolar y más del $80 \%$ de los actos de abuso que no son reportados por los maestros, ya que el bullying es acto de acoso muy violento. El bullying a nivel escolar se ha transformado en una alerta roja para los profesores y directores de las escuelas ellos están muy interesados en mejorar el ambiente educativo, sin embargo, es de vital importancia conocer las causas que orillan a los jóvenes a tomar actos de acoso con los demás estudiantes para finalmente poder intervenir con procesos educativos que impulsen un cambio. Según la psicóloga y periodista Henar Senovilla las causas al entorno educativo que pueden motivar el acoso entre estudiantes son incalculables, aunque una de ellas puede estar motivada por una cuestión de ideologías, racismo o preferencias sociales. El bullying también se da generalmente por los problemas en el hogar o también cuando un niño se cría sin una figura paterna o porque conviven con personas que lo agreden tanto física como verbalmente. 
Figura N 3 Causas del Bullying.

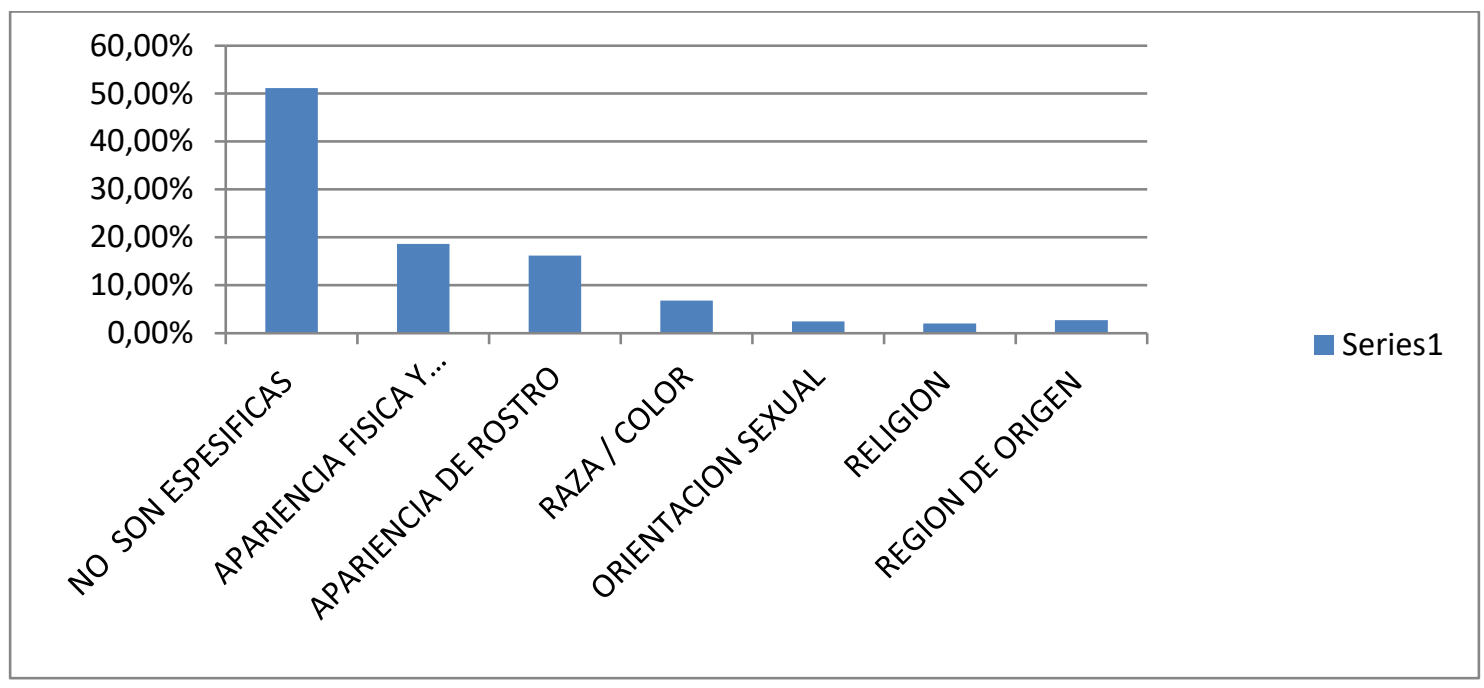

Fuente: Elaborada propia

\section{Criterio.}

En mi opinión las causas o factores del bullying son provocados en los centros educativos estos suelen ser por problemas personales, familiares y escolares nos dice que por lo general el acosar se ve superior a los demás o también porque cuenta con el apoyo de los demás atacantes, o porque el acosado es alguien con muy poca capacidad de responder a todas las agresiones, en las mayorías de los casos el agresor se siente satisfecho de ver sufrir al acosado y que este la esté pasando muy mal solo así se sentirá satisfecho. Aparte de esto el trato que se les da a los alumnos es muy importante. También nos dice que la falta de respeto, la humillación, las amenazas o la exclusión del personal docente de las escuelas o colegios conlleva a que los alumnos reacciones con violencia o en otros casos con agresión.

\section{Estrategias para eliminar el bullying.}

Según los autores (Mora, 2006), (Students, 2014) \& (Vargas, 2017), el bullying o acoso escolar constituye una forma verbal física o escrita que genera un daño en otro individuo, si bien no existen recetas mágicas para combatir el acoso escolar hay un grupo de especialistas que creen que la problemática podría ser combatida mediante las siguientes medidas, la buena educación desde la infancia, informar a los padres, capacitar a los profesores, acabar con el silencio. También se elaboró una serie de consejos en caso de sospechar que un niño está siendo hostigado por sus otros compañeros, Identificar conductas de hostigamientos en la escuela, armar reglamentos de convivencia entre los alumnos, fomentar la ayuda entre pares, diseñar métodos de resolución de conflictos entre pares, corregir al alumno ante cualquier conducta de exclusión de un par, amonestar ante cualquier conducta agresiva ya sea física o verbal, fomentar actividades de convivencia, también poner una buena supervisión en las 
horas del recreo, crear un sistema para que los alumnos para que puedan reportar los casos de hostigamiento, armar reuniones entre padres y profesores para resolver los conflictos que se dan en las escuelas o colegios estas y otras estrategias son de muy importancia que debemos poner en práctica para que los niños y niñas y adolescentes no sean víctimas de acoso escolar.

\section{Criterio.}

En mi opinión se ha venido buscando mucha información sobre el bullying o acoso escolar, nos podemos dar cuanta que la mayoría de los medios de comunicación lanzan a diario imágenes de niños y jóvenes peleando violentamente, es por esto que debemos buscar muchas estrategias para eliminar el acoso escolar ya que esto es algo que afecta física, psicológica y mental mente. Prevenir y erradicar el acoso escolar requiere del compromiso de diversos involucrados que permitan crear un ambiente seguro en el que los niños y jóvenes puedan aprender a no ser agredidos existen estrategias para que los profesores puedan estar alertas: estar informado y alerta, los maestros y administradores deben enfatizar que informar no es delatar, otra de las estrategias también es la participación de estudiantes con sus padres, crear expectativas positivas sobre el comportamiento de estudiantes y adultos, con todas estrategias podemos evitar que el acoso escolar continúe en las escuelas ser

\section{Conclusiones}

- La investigación realizada a contribuido de manera muy importante que para identificar y resaltar los aspectos que giran al entorno de la problemática denominada Bullying, sobre todo aquellos que ignoran o desconocen en tema, llámense padres de familia, docentes, e incluso los mismo alumnos.

- Esto también nos deja muchas cosas que reflexionar y muchas otras las ha reforzado, como lograr identificar a un agresor o una víctima por medio de la observación, sin la necesidad de realizar un psicoanálisis.

- Es muy importante plantear que en bullying no es superior, la violencia solo conduce a más violencia y que el hecho de ser más fuerte no te hacer mejor, o tener ciertas características como usar lentes o tener sobrepeso, o ser aplicado en la escuela no te hace inferior a los demás.

- Establecer los objetivos de cada caso y ordenarlos según la importancia que se esté suscitando para que así podamos tomar una muy buena decisión y poder solucionarlo y obtener buenos resultados

\section{Referencias bibliográficas.}

Aguirre, G. (2014). Normese en el codigo de la niñez y adolescencia el acoso escolar (bullying) En el ecuador. Loja: MED. 
Aviles, J. (2015). Diferencias de atribución causal en el bullying entre sus protagonistas. 2015: Redalyc.

Casilla, S. (2008). Maltrato entre pares o "bullying". Scielo , 1.

Cava, M. (2012). Factores de riesgo y consecuencias del cyberbullying en un grupo de adolescentes. Mexico: Redalyc.

Cerezo, F. (2002). El bullying y su relación con las actitudes de socialización en una muestra de adolescentes. Dialnet, 1.

Cusot, G. (2012). Bullying en el Ecuador Comunicación para el mercadeo social. Quito: USFQ.

Garcia, X. (2010). Factores relacionados con el acoso escolar (bullying) en los adolescentes . Barcelona: Gaceta Sanitaria.1

Lossi, A. (2015). Causas del bullying:. Sau Pablo: Latino-Am. Enfermagem.

Medina, V. (2015). Acoso escolar. Guiainfantil.

Meglino, J. (8 de junio de 2018). ONG Internacional Bullying Sin Fronteras. Recuperado el 2 de noviembre de 2018, de ONG Internacional Bullying Sin Fronteras: https://bullyingsinfronteras.blogspot.com/

Merchan, A. (11 de Enero de 2018). Estadísticas de Bullying . Recuperado el 2 de Noviembre de 2018, de Estadísticas de Bullying : https://bullyingsinfronteras.blogspot.com/2017/05/estadisticas-de-bullying-enespana-mayo.html

Mora, J. (2006). Estrategias para eliminar el bullying. Sevilla: APCS.

Morales, H. (2008). Violencia juvenil. Pepsic.

Pando, M. (2012). Analzis confirmatirio de violencia en ecuador . Scielo , 1.

Portero, L. (2007). El "bullying" y otras formas de violencia adolescente. Scielo , 1.

Rubio, M. (2007). Pobreza, educación, mujeres y violencia juvenil. Ideas , 1.

Sanchez, A. (14 de octubre de 2018). Estadísticas de Bullying en América Latina. Primer Estudio Internacional. 2018. Recuperado el 2 de noviembre de 2018, de Estadísticas de Bullying en América Latina. Primer Estudio Internacional. 2018.: 
https://bullyingsinfronteras.blogspot.com/2018/02/estadisticas-de-bullying-enamerica.html

Sanchez, N. (2012). Factores de riesgo y consecuencias del cyberbullying en un grupo de adolescentes. Mexico: Medigraphic.

Santiago Casilla. (2008). Maltrato entre pares o "bullying". Scielo , 1.

Students, T. (23 de Juliio de 2014). estrategias para combatir el acoso escolar. Recuperado el 2 de noviembre de 2018, de estrategias para combatir el acoso escolar: http://noticias.universia.edu.pe/en-portada/noticia/2014/07/23/1101019/bullying-4estrategias-combatir-acoso-escolar.html

Vargas, B. (17 de Octubre de 2017). estrategias para prevenir el bullying en el salón de clases. Recuperado el 2 de noviembre de 2018, de estrategias para prevenir el bullying en el salón de clases: https://noticias.universia.net.co/cultura/noticia/2015/10/23/1132740/5-estrategiasprevenir-bullying-salon-clases.html

Wielandt, G. (2005). Vilencia juvenil en centroamerica . Santiago de Chile: Casa del libro.

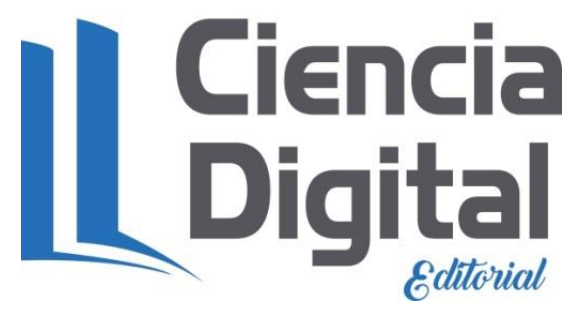


Para citar el artículo indexado.

Velasteguí López, P. (2019). Efectos del bullying en los adolescentes. ConcienciaDigital, 2(2), 6-16. https://doi.org/10.33262/concienciadigital.v2i2.940

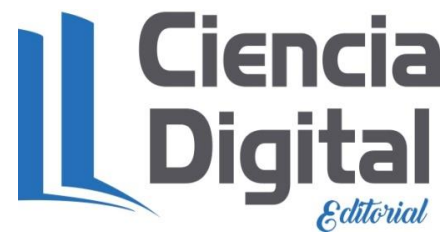

El artículo que se publica es de exclusiva responsabilidad de los autores y no necesariamente reflejan el pensamiento de la Revista Conciencia Digital.

El articulo queda en propiedad de la revista y, por tanto, su publicación parcial y/o total en otro medio tiene que ser autorizado por el director de la Revista Conciencia Digital.

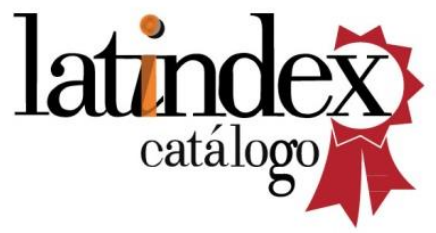

Conciencia

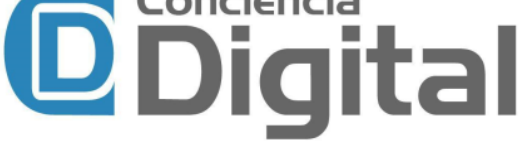

